\title{
Author Correction: Invasion of white matter tracts by glioma stem cells is regulated by a NOTCH1-SOX2 positive-feedback loop
}

Jun Wang, Sen-Lin Xu, Jiang-Jie Duan, Liang Yi, Yu-Feng Guo, Yu Shi, Lin Li, Ze-Yu Yang, Xue-Mei Liao, Jiao Cai, Yan-Qi Zhang, Hua-Liang Xiao, Li Yin, Hao Wu, Jing-Na Zhang, Sheng-Qing Lv, Qing-Kai Yang @ , Xiao-Jun Yang, Tao Jiang, Xia Zhang, Xiu-Wu Bian (1) and Shi-Cang Yu (D)

Correction to: Nature Neuroscience https://doi.org/10.1038/s41593-018-0285-Z, published online 17 December 2018.

In the version of this article initially published, the $x$-axis labels in Fig. $8 \mathrm{f}-\mathrm{h}$ should have read "Days after surgery" and the first sentence of the Methods section should have read "The human glioma cell line U87 was derived from the American Type Culture Collection. The murine glioma cell line GL261 was gifted by Z. Wang (Beijing Institute of Transfusion Medicine, Beijing, China). The cell lines were verified through STR testing (Beijing Microread Genetics Co., Ltd., Beijing, China)." The errors have been corrected in the HTML and PDF versions of the article.

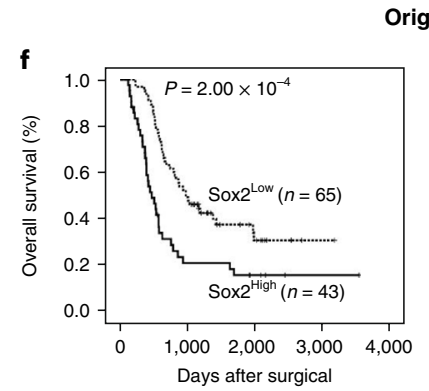

Original
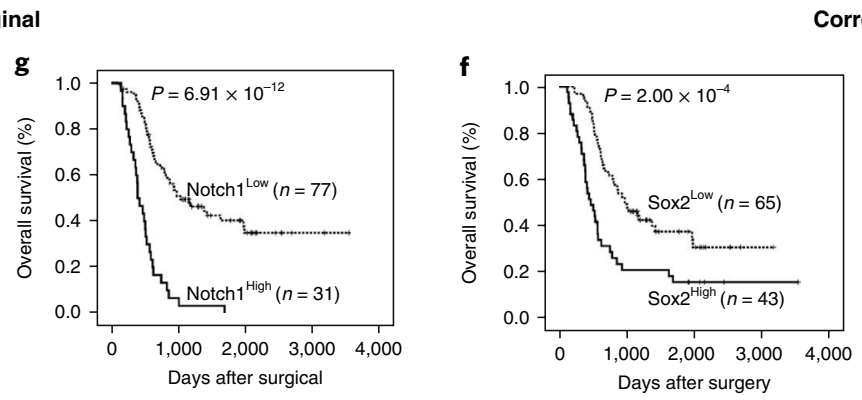

Corrected
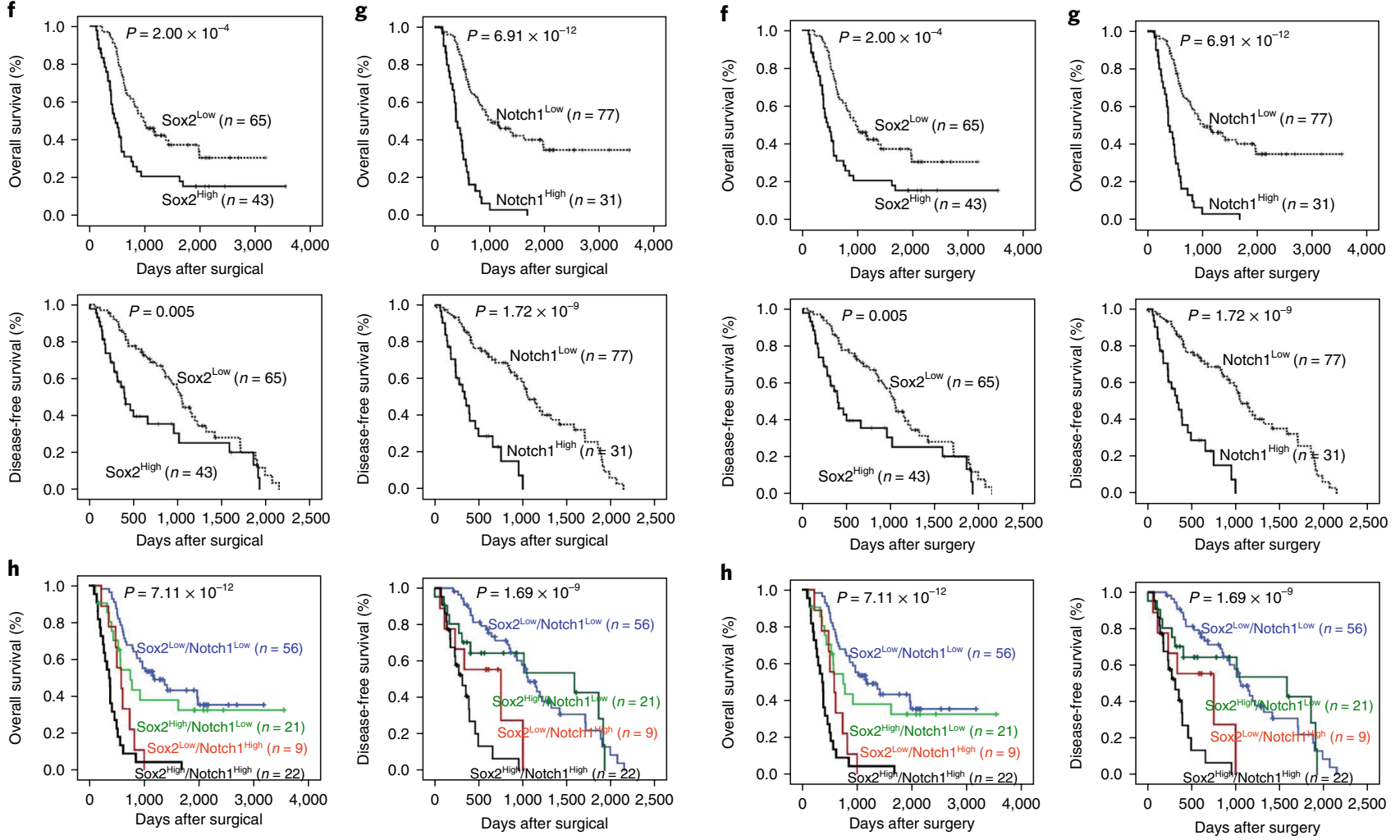

Fig. 8 | Original and Corrected.

Published online: 22 March 2019

https://doi.org/10.1038/s41593-019-0388-1

\section{Author Correction: Neural computations of threat in the aftermath of combat trauma}

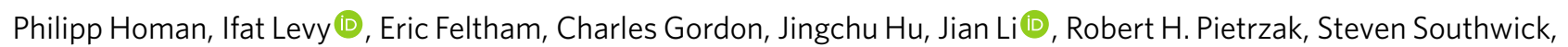
John H. Krystal, Ilan Harpaz-Rotem (1) and Daniela Schiller (D)

Correction to: Nature Neuroscience https://doi.org/10.1038/s41593-018-0315-x, published online 21 January 2019.

In the version of this article initially published, graphs in Fig. 4 were labeled incorrectly. The figure has been replaced in the HTML and PDF versions of the article. In the PDF version of this correction, original and corrected figures may appear on the next page. 